\title{
Depressive Disorders in Patients with Epilepsy: Underdiagnosed and Appropriately Managed?
}

\section{Taoufik Alsaadi $^{1^{*}}$ and Tarek M Shahrour ${ }^{2}$}

${ }^{1}$ Department of Neurology, Sheikh Khalifa Medical City (SKMC), UAE

${ }^{2}$ Department of Psychiatry, Sheikh Khalifa Medical City (SKMC), UAE

"Corresponding author: Taoufik Alsaadi, Department of Neurology, Sheikh Khalifa Medical City (SKMC), UAE, Tel: +97128190000; E-mail: talsadi@skmc.ae

Rec date: Jan 23, 2015; Acc date: Feb 12, 2015; Pub date: Feb 16, 2015

Copyright: (C) 2015 Alsaadi T, et al. This is an open-access article distributed under the terms of the Creative Commons Attribution License, which permits unrestricted use, distribution, and reproduction in any medium, provided the original author and source are credited.

Abstract
Epilepsy is a complex disorder that is commonly associated with additional brain dysfunction, social isolation, and
vocational difficulty. Each of these factors may contribute to the increased prevalence of psychiatric disorders in
epilepsy. Yet, epidemiological data suggest that depression is the most prevalent disorders among all psychiatric
illness in epilepsy. Likewise, several emerging data have explored several multifactorial etiologies of depression in
epilepsy. These include underlying genetic, neurochemical, anatomical, neurologic, and iatrogenic factors.
Furthermore, clinical investigations have consistently demonstrated that depression has a large impact on subjective
health status. In patients with recurrent seizures, depression appears to have a stronger association with QOL than
does seizure rates. Unfortunately, however, a significant proportion of patients with epilepsy and depression are not
diagnosed nor offered the appropriate treatment. Current treatment recommendations for depression in epilepsy are
similar to those for otherwise neurologically normal depressed patients, emphasizing the role of SSRI, but certain
antidepressants should be used with caution. Ongoing studies are attempting to define optimal treatment strategies,
and more definitive data to guide clinical management is expected to become available in the near future.

Keywords: Depression; Seizures; Temporal Lobe Epilepsy (TLE); Quality of Life (QOL); Serotonin Reuptake Inhibitors (SSRIs)

\section{Abbreviations: \\ AED: Antiepileptic Drugs; BDI: Beck Depression Inventory; MTS: Mesial Temporal Sclerosis; NDDI-E: Neurological Disorders Depression Inventory for Epilepsy; PHQ-9: Patient Health Questionnaire nine-item depression scale; PWE: Patients with Epilepsy; QOL: Quality of Life; SSRIs: Serotonin Reuptake Inhibitors; SNRI: Serotonin and Epinephrine Reuptake Inhibitors; TLE: Temporal Lobe Epilepsy; TCA: Tricyclic Antidepressant}

\section{Introduction}

Epilepsy is a chronic condition that may be associated with several other neurological disorders; stroke, migraine, and psychiatric disorders are the most frequent comorbid disorders in PWE. The term comorbidity is used to describe greater than coincidental association of two or more conditions in the same individual [1].

Epilepsy has been associated with increased risk of psychiatric disorders. Although the incidence and prevalence rates of psychiatric comorbidities vary widely among studies from $12 \%-41 \%$, this variation is largely due to methodological differences among the studies; selection bias for the population under study, diagnostic methods used, AED numbers and dosages are some of the confounding factors that could have an impact on the prevalence rates [2-5] Epilepsy has also been associated with increased risk of suicide, even after adjustments for various factors known to pose a risk for suicide in the general population [6-9].

The most frequent psychiatric diagnoses reported in people with epilepsy include psychoses, neuroses, mood disorders (DSM-IV axis I disorders), personality disorders (DSM-IV axis II disorders), and behavioral problems. Among these, mood disorders are the most frequent psychiatric comorbidity in PWE with a prevalence of depression estimated between $11 \%$ and $60 \%$ in patients with recurrent seizures $[10,11]$. Indeed, it is well established that one of every three PWE will experience a depressive disorder in the course of their life, often associated with anxiety symptoms or a full blown anxiety disorder.

\section{Worldwide Prevalence of Depression in Epilepsy}

Based on the available worldwide epidemiological studies, the prevalence of depression ranges from 11 to $60 \%$ in patients with recurrent seizures and from 3 to $9 \%$ in patients with well-controlled seizures. Mendez et al. [11] used the Hamilton Depression Rating Scale in 175 consecutive patients in an outpatient epilepsy clinic, concluding that $55 \%$ met criteria for depression. In a communitybased study that used the Hospital Anxiety and Depression Scale, Jacoby et al. [12] observed, in a well-designed survey, that $21 \%$ of 168 patients with recurrent seizures were depressed; whereas, only $9 \%$ of controlled patients had significant symptoms of depression. On the other hand, in a primary care setting, O'Donoghue et al. [13] used the same scale to demonstrate that, out of a group of 155 patients in the United Kingdom, 33\% with recurrent seizures and 6\% of those in remission had probable depression. Similarly, a study by Seyal et al. [14] using PHQ-9 found that $30 \%$ of their patients attending a specialized epilepsy clinic at the University of California at Davis, USA have scored in the depressed range. Likewise, a recently submitted work in UAE has drawn similar conclusions [15] In fact, despite several methodological variability among various studies, depression is consistently found to be up to 10 times more prevalent in association with uncontrolled epilepsy than in the general population. Finally, a recently published meta-analysis of all well designed studies of 
depression in PWE concluded that the risk of depression increases several folds in patients with epilepsy, when compared to the general population [16]. The findings from all these studies underscore the importance of depression in epilepsy and the need to effectively screen and treat depressive disorders.

\section{Impact of Depression on Health Related Status}

Several studies have studied the implication of depression on health related quality of life in PWE. Ettinger et al. [2] has utilized the household panel maintained by the National Family Opinion to study depression and QOL in persons with epilepsy, asthma, and healthy controls. The response rate for the survey was approximately $50 \%$ in each group, with a total of 1532 responses. PWE were significantly more likely to score in the depressed range on the Center for Epidemiological Studies Depression Scale (CES-D) (37\%), than were those with asthma $(28 \%)$ or healthy subjects (12\%). Although nearly half of the group with epilepsy had not had a seizure during the past year, suggesting that the sample represented the less severe aspect of the spectrum of epilepsy, the mean scores on the Short Form-36 scales for role limitations, emotional wellbeing, and social wellbeing were significantly worse than the asthma group. On the other hand, none of the scales was lower for asthma. In another cross sectional study, Beghi et al. [17], compared depression severity across disease states in a study of epilepsy, type I diabetes mellitus, and community controls. Fifty-five patients with idiopathic or cryptogenic epilepsy were compared with age and sex matched subjects with type I diabetes or persons donating blood in the local medical clinic. Epilepsy subjects with any structural brain abnormality were excluded from the study, and only $37 \%$ reported a seizure within the past year, reflecting less form of severe epilepsy, similar to, the study of Ettinger et al. [2] Thirty-four percent of epilepsy patients scored in the depressed range compared with $27 \%$ of type I diabetes patients and $7 \%$ of blood donors.

\section{The Importance of Identifying Depression in PWE}

The impact of depression on QOL in PWE has been well described in five studies involving patients with pharmacoresistant epilepsy. They demonstrated that depression is the most powerful predictor of health related quality of life, even after controlling for seizure frequency, severity, and other psychosocial variables [18-22]. Furthermore, a recent study has found that mood disorders are strongly and, independently, associated with worse QOL after epilepsy surgery [23]. On the other hand, several studies have demonstrated the increased incidence of suicide in patients with depression and epilepsy. In a review of 11 studies, Harris and Barrowclough [24] have estimated the overall suicide rate in people with epilepsy to be five times higher than in the general population and 25 times greater for patients with complex partial seizures of temporal lobe origin. In a review of the literature, Jones et al. [6] identified a lifetime average suicide rate of $12 \%$ in people with epilepsy compared to $1.1 \%$ to $1.2 \%$ in the general population.

Moreover, other studies have clearly shown that depression in people with epilepsy has significantly increased the healthcare costs associated with the management of the seizure disorder. Cramer et al. [25] found that patients with untreated depression used significantly more health resources of all types, independent of seizure type or latency. Likewise, mild-to-moderate depression was associated with a two-fold increase in medical visits compared with nondepressed controls, while severe depression was associated with a four-fold increase.

Additionally, and more importantly perhaps, depression in PWE might be a marker of "bad" epilepsy. A well designed study suggests that the presence of depression in PWE may predict a failure to respond to pharmacotherapy with AEDs in patients with newly diagnosed epilepsy. Of a cohort of 890 patients with newly diagnosed epilepsy, Hitiris et al. [26] found that a prior history of depression is associated with at least 2.5 times risk of not responding to AEDs over 5 years of follow up. Likewise, the presence of depression prior to epilepsy surgery, may also serve as an independent marker for worse postsurgical outcome following anterior temporal lobectomy (ATL), as shown by the study of Kanner et al that included 100 patients with a mean follow up of 4 years [27]. These studies could support the hypothetical believe that depression can be considered as a marker for "difficult to control" epilepsy.

Finally, and on a separate note, it is worth mentioning that epilepsy may have an extensive impact on patients' physical, psychological, and social functioning (i.e., QOL). QOL in PWE is affected by several components, one of which is related to comorbid mood disorder, where high rates of anxiety, depression, and low self-esteem have been reported among these patients. Furthermore, they are more likely to be under- or unemployed, to be at great risk of educational achievements, and face restrictions on driving and social stigma, and, for women, they are at fear of lower rates of marriage and having children. Other factors to be addressed in PWE are broad; seizure control status, burden of AEDs in relation to dosages and numbers, other chronic comorbidity conditions that may negatively and, independently, affect the QOL in these patients and would also affect the choice of AED therapy.

\section{Etiologies of Depression in Epilepsy}

The perception that depression is a "normal" response to having a chronic condition such as, epilepsy has long been held, by both patients and physicians, but is no longer acceptable or valid. Instead, several emerging data have explored other multifactorial etiologies of depression in epilepsy. These include genetic, neurochemical, anatomical, neurologic, and iatrogenic factors.

\section{The role of genetics}

The role of genetics in the etiology of depression in epilepsy is suggested by the fact that a family history of depression is quite common among depressed patients with epilepsy. More than half of these patients have been reported to have family histories of psychiatric illness, usually mood disorders [28].

\section{The role of neurotransmitters}

It appears that epilepsy and depression may share common pathogenic mechanisms involving decreased serotonergic, noradrenergic, dopaminergic, and GABAergic activity, which has been shown to take part in the kindling process of seizure foci, exacerbating seizure frequency in some animal models [29]. Studies of neurotransmitter activity in both epilepsy and depression suggest that the occurrence of one disorder may facilitate the development of the other, and vice versa [30]. As a matter of fact, several imaging studies utilizing interictal PET using different ligands, have consistently shown some degree of decreased 5-HT1A binding in the mesial structures, raphe nuclei, thalamus, and cingulate gyrus [31-33]. In a 
Page 3 of 9

study of 45 patients with TLE, Theodore et al demonstrated an inverse correlation between increased severity of symptoms of depression identified on the BDI and 5HT1A receptor binding at the ipsilateral hippocampus to the seizure focus and to a lesser degree at the contralateral hippocampus and midbrain raphe [34]. These changes in 5 HT 1 A receptor binding are quite similar to those identified in PET studies of patients with primary major depressive disorders (MDDs). To further support this shared pathogenic mechanisms, three casecontrol population based studies have shown that a history of depression was associated with several fold increased risk for developing new onset epilepsy among cases than among controls [35-37]. More recently, a population-based case-control study from Stockholm with new onset unprovoked seizures assessed the risk of developing unprovoked epileptic seizures before and after hospitalization for a psychiatric diagnosis. The age-adjusted odds ratio for unprovoked seizures was 2.5 after a hospital discharge diagnosis for depression, 2.7 for bipolar disorder, 2.3 for psychosis, 2.7 for anxiety disorders, and 2.6 for suicide attempts [38].

Although the data from all these case-controlled suggest a bidirectional relationship among depression and epilepsy, it does not necessarily, however, suggest that depression causes epilepsy or vice versa. Rather, it may support the point towards the existence of common pathogenic mechanisms operant in both conditions which facilitate the development of one disorder in the presence of the other. Interestingly, many centuries ago, Hippocrates $400 \mathrm{BC}$ had suggested this type of bidirectional relationship when he wrote, "Melancholics ordinarily become epileptics, and epileptics melancholics: what determines the preference is the direction the malady takes; if it bears upon the body, epilepsy, if upon the intelligence, melancholy". Galen at $200 \mathrm{AD}$ had further reconfirmed the organic cause of depression [39].

\section{Neuroanatomical factors}

Changes in common brain structures have been identified in patients with primary major depressive and bipolar disorders and in PWE, including atrophy of temporal- and frontal-lobes. These changes have been identified with the use of high-resolution MRI and volumetric measurements of the amygdala, hippocampus, entorhinal cortex, temporal, lateral neocortex, as well as of the prefrontal, orbitofrontal, and mesial-frontal cortex, and to a lesser degree, of the thalamic nuclei and basal ganglia [40]. It is, therefore, not surprising that PWE whose seizure foci are in temporal and frontal lobes have a higher prevalence of depression. Furthermore, hippocampal atrophy/ sclerosis (HS) which is a hallmark of mesial temporal lobe epilepsy is a common finding in patients with depression and, according to some studies, correlate with both the severity and duration of the depressive state [41]. Furthermore, Quiske et al. [42] examined the association of MTS on MRI with BDI scores in 60 patients with TLE. Mean depression scores were significantly higher in patients with MTS, independent of the lateralization of MTS. The investigators suggested depression as "a good indicator of MTS, but not vice versa."Moreover, a study by Gilliam has shown a correlation of the degree of MR spectroscopic abnormalities in the ipsilateral hippocampus in patients with TLE and the severity of depression as measured by BDI [43]. In addition, additional studies suggest that the presence of HS is a risk factor for developing depression in PWE when treatment is initiated with AEDs with depressive properties, such as topiramate, and levetiracetam $[44,45]$.

\section{The role of seizure related factors}

Seizure types have been shown, in some studies, to correlate with depression. Depression is more commonly encountered in patients with complex partial seizures, particularly those of temporal lobe origin [15]. Others have looked at the possibility that depression might be associated with laterality of seizure focus. Most studies have found that depression is more common in those with left-sided foci [46]. Furthermore, a recent work by Theodore et al suggests that depression, when measured by BDI sores, is more likely to occur in patients with right TLE and left MTS, as compared with, left TLE without depression [47].

\section{The role of iatrogenic factors (drug related)}

Among the most important factors contributing to the risk for depression in PWE are those associated with medication side effects. A number of drugs and drug classes have been implicated in the etiology of depression, including some AEDs, such as barbiturates, levetiracetam, vigabatrin, and topiramate. These drugs and, especially in patients susceptible to psychiatric illness, (e.g., history of a prior psychiatric illness and/or family psychiatric illness) can be clearly associated with behavioral changes and depression [45]. Other investigators have related depression to the rapid withdrawal of an AED with mood stabilizing properties, such as, carbamazepine, oxcarbazepine, and lamotrigine [48]. Likewise, and as an example of drug related depression, the addition of AEDs with enzyme-inducing AEDs (e.g., phenobarbital, primidone, phenytoin, carbamazepine, high-dose topiramate, and oxcarbazepine) in patients taking antidepressant drugs that had yielded a remission of a depressive episode. The increased clearance of the antidepressant drug results in lower serum concentration and potential loss of efficacy. Another, uncommon, but well documented example is the occurrence of de novo depression following ATL. Interestingly, this risk occurs independently of postsurgical outcome [49].

\section{Geschwind syndrome and TLE}

As an example of the close relationship between psychiatric issues and epilepsy, Geschwind and Waxman were the first to describe a specific set of personality alterations in PWE which was later called Geschwind syndrome [50]. This syndrome has been described in patients with TLE and is characterized by sexual behavioral disorders, hyperreligiosity, hypergraphia, and viscosity. The existence of this syndrome, as a specific personality disorder, in PWE is not accepted unanimously and has led to considerable debate [51,52]. The evidence for its existence is largely empirical, and following Benson [53]. The strongest support so far stems from the many clinicians who have described and attempted to manage patients with epilepsy and these personality features. It has also been described in a subgroup of patients with TLE with bilateral hippocampal atrophy [54]. Its occurrence, however, in patients with unilateral hippocampal atrophy is very questionable.

\section{Clinical manifestations}

Depressive symptoms can present according to the temporal relation to the seizures occurrence into ictal (the depressive symptoms are a clinical manifestation of the seizure, a rare form of depression in $\mathrm{PWE}$ ), periictal (symptoms precede and/or follow the seizure occurrence), and interictal (symptoms occur independently of the seizure occurrence). Interictal depression is the most frequently 
"recognized" type of mood disorder and can present differently among PWE. Major depression, bipolar disorder, dysthymic disorder, and minor depression are all well described in PWE. Nevertheless, a significant percentage of patients present an atypical clinical picture that fails to meet any of the DSM Axis I categories, which lead Blumer to coin the term "interictal dysphoric disorder" to refer to this type of depression in epilepsy with its prolonged and interrupted depression free course that is often associated with anhedonia, hopelessness, helplessness, anergia, pain, and insomnia [55]. In a study by kanner et al, symptoms of depression mimicked dysthymic disorders in 69 of 97 consecutive patients (70\%); the interrupted nature of these symptoms accounted for the failure to meet DSM-IV criteria of dysthymic disorder [29].

The underlying etiology of this interrupted course is not well explained. Conceivably, it is possible that breakthrough seizures in PWE might interrupt the course of depression and improve the depressive symptoms episodically and hence the interrupted course of the disease [56]. On the other hand, the phenomenon of "forced normalization" may also explain in part the interrupted nature of the disease. This phenomenon consists of the appearance of psychiatric disorders associated with the cessation of epileptic seizures [57]. Having said that, the relationship between depression and epilepsy is far from being that simple. Indeed, a recent study showed, in contrast to the forced normalization concept, a significant improvement in depression and anxiety symptoms in patients with refractory epilepsy following epilepsy surgery, especially in those who became seizure-free [49].

\section{Screening for depression}

Despite its high frequency and great impact on the QOL and care for PWE, depression remains undertreated and unrecognized in a significant number of PWE. Kanner et al. determined that $63 \%$ of patients with spontaneous depression and $54 \%$ of patients with an iatrogenic depression were symptomatic for more than 1 year before treatment was initiated [24]. While the clinical manifestations of depression in people with epilepsy can be atypical, the most frequent cause for this underdignosis is the failure of clinicians to inquire about the symptoms, and for patients and families to report them, assuming that it is quite normal to have them in the setting of this chronic disease. In a survey of neurologists, Gilliam found that $80 \%$ do not routinely screen for depression in patients with epilepsy [58]. This last figure suggests that in any busy neurology practice, where the major focus is seizure control, it is very likely that symptoms of depression may be easily missed.

Several tools have been used to screen for depression in PWE; some of which are time consuming in a busy clinic setting. A 6-item questionnaire referred to as NDDI-E has been developed and validated to screen for depression. It takes less than 3 minutes to fill out at the office and has a sensitivity and specificity of $90 \%, 81 \%$, respectively [59]. A score of 14 and higher is suggestive of the possibility of a major depressive disorder and can serve as an alarming sign to carry out a more in-depth evaluation. A recent study enrolled 266 PWE at a specialized neurologic epilepsy service in London and compared verbal self-report and visual analog (VAS) screening methods for depression. These included two generic depression scales (Hospital Anxiety and Depression Scale [HADS], BDI-II, one epilepsy specific scale [NDDI-E]) and one new visual-analog scale (Emotional Thermometers [ET]). The authors conclude that the six-item NDDI-E or seven-item HADS-D should be considered if a conventional scale is preferred and that the revised ET4 be considered if a visual-analog method is required [60]. Clearly, the use of these screening instruments for psychiatric research on epilepsy must be followed by structured psychiatric interviews designed to establish a DSM-IV-TR diagnoses, which would permit regular rescreening to yield meaningful data on changes in severity of symptomatology. In support of using these instruments, a recently well designed study evaluated the accuracy of, and the operating characteristics of PHQ-9 for depression screening in PWE and concluded that PHQ-9 is an efficient and nonproprietary depression screening instrument with excellent accuracy validated for use in PWE [61].

\section{AEDs and suicidal risk}

To highlight the seriousness and complexities of psychiatric problems associated with AED use, a recent meta-analysis has linked AED use with increased suicide risk [62] The meta-analysis encompassed a total of 43,892 patients who were treated for epilepsy, psychiatric disorders and various pain disorders. The FDA concluded and based on that meta-analysis that, with the exposure to AEDs, the risk for suicidality was increased by a statistically significant 1.80 fold. Suicidality occurred in 4.3 per 1000 patients treated with AEDs in the active arm, compared with 2.2 per 1000 patients in the comparison arm. The use of AEDs was associated with a higher risk for suicidality in PWE (odds ratio: 3.53; 95\% CI: 1.28-12.10) than in patients with psychiatric disorders (odds ratio: 1.51; 95\% CI: 0.95-2.45) or other disorders (odds ratio: 1.87; 95\% CI: 0.81-4.76). Despite the aforementioned caveats for linking AED use to any psychopathology, and the methodological issues associated with the meta-analysis [63], a detailed evaluation questioned the validity of its findings owing to several methodological problems [64]. Furthermore, this risk is insignificant if, compared with the average lifetime incidence of suicidality, in PWE attending epilepsy clinics [6]. As a matter of fact, and since the FDA warning, at least three large studies have attempted to clarify whether AEDs are associated with an increased suicidal risk [65-67]. The data from these studies yielded contradictory results. A study by Gibbons et al. in patients with bipolar disorders taking AEDs, concluded that AEDs, as a class, are not associated with an increased risk of suicidality [65]. On the other hand, a study by Patorno et al. found a significantly higher risk of suicidality and violent deaths with certain AEDs, such as lamotrigine, oxcarbazepine, gabapentin and tigabine. Unlike the finding from the FDA meta-analysis, topiramate was not associated with that risk [67]. Based on all the above reviews and studies, we can propose that several AEDs, but not all, as suggested by the FDA warning, might be associated with psychiatric adverse events, which may lead to suicidal ideation and behavior, keeping in mind that the rates of completed suicide and suicidal attempts are, fortunately, rare. It is worth mentioning, however, that given the relatively high prevalence of comorbid mood and anxiety disorders in PWE [8], as well as the increased suicidal risk in PWE [5], clinicians should be alerted to screen patients for both conditions, independently of whether, AEDs have any influence on suicidal risk. Specifically, clinicians should be extremely alert to the high risk in patients with a current and or past psychiatric history [6].

\section{Treatment options}

Despite its relatively high prevalence and significant impact on management, the treatment of depression in PWE remains an, "unexplored territory." Indeed, a significant percentage of PWE and depression are under-recognized and under -treated. One reason for 
this includes the fear that seizures are exacerbated by antidepressants. To that extent, the treatment of these patients has been, for all intents and purposes, empiric, based on the untested assumption "that patients with depression and epilepsy should respond to antidepressant drugs in the same manner as depressed nonepileptic patients." In fact, there has only been one small double-blind, placebocontrolled study published till recently that included 42 patients. It compared the efficacy of mianserin, amitriptyline, and placebo to treat major depression in PWE in three treatment arms. At the end of 6 weeks of treatment, no significant differences in outcome were observed between the groups. It is very clear that the small sample size of that trial limits the draw of any meaningful conclusions [68].

Similarly, and in a very recently published Cochrane review on this topic, further confirms the limitations of the evidence on the efficacy of antidepressants in treating depressive symptoms associated with epilepsy. As a matter of fact, in that systematic review, only one small RCT have met the inclusion criteria and demonstrated a statistically significant effect of venlafaxine on depressive symptoms. The authors have urgently, and, correctly so, called for further comparative clinical trials of antidepressants and psychotherapy in large cohorts of patients with epilepsy and depression [69]. On that note, and to further show the complexity of this matter, it is of equal importance to keep in mind that in patients with depression, but without any other comorbid condition, a meta-analysis performed by NICE of all the placebo controlled trials of SSRI did not find any clinically significant difference between placebo and SSRIs arms [70].

\section{Treatment strategies}

Before starting a patient on antidepressant drug therapy, it is important to realize that AEDs have a range of beneficial and adverse psychotropic effects. Some AEDs can be effective as treatments for mania and bipolar depression and as mood stabilizers in bipolar and schizoaffective disorders on one hand, and may in some patients on the other hand, cause depressive symptoms [71]. Obviously, establishing the causal role of any particular AED in regard to an affective symptom is quite complex, as it may be confounded by the type of epilepsy, other co-occurring medication exposures, preexisting psychopathology, cognitive deficits, the impact on seizure activity (e.g. a marked reduction in seizures may have either beneficial or it may carry adverse effects on mood) and the pharmacokinetic effects. Furthermore, the characterization of psychological symptoms is often poor; for example, many studies record 'agitation' and 'irritability', ill-defined symptoms that may stem from anxiety or other causes; similarly, 'apathy' or 'tiredness' may stem from depression or other causes. Therefore, and as previously discussed in the section of "iatrogenic causes of depression", it is widely recommended to rule out the following potential causes for depression before treatment with antidepressant is initiated 1) the depressive episode followed the discontinuation of an AED with mood stabilizing properties, such as carbamazepine, valproic acid, or lamotrigine, especially in patients with a past and/or a family psychiatric history, as these AEDs may be keeping an underlying mood disorder in remission [48]. In such a case, reintroduction of that AED or of another mood-stabilizing agent may be sufficient to reach a euthymic state and 2) the depressive episode followed the introduction or dose increment of an AED with known negative psychotropic properties, such as Phenobarbital, topiramate, vigabatrin, and levetiracetam. Depressive episodes in these cases may be dose-related, with some (e.g., barbiturates, benzodiazepines). In such cases, lowering the dose of AED, without compromising seizure control, if possible, may result in symptom remission. On the other hand, depression may occur de novo in patients taking levetiracetam, regardless of the dose, the patient is taking, i.e, lowering the dose of the drug may not result in symptoms remission. In these patients, discontinuation of the medication or, in case levetiracetam was the most effective AED for that patient, the addition of an antidepressant should be considered. Other AEDs, however, such as topiramate can cause depression as result of rapid titration of the medication [72]. In such cases, slowing of the titration rate of the culprit AED may result in symptom remission. However, if an AED to be discontinued, it is important to remember that expert consensus has proposed a preferred approach to transitional alternative monotherapy, recommending that an existing baseline AED be tapered only after a presumably efficacious dose of the new adjunctive AED is reached. Initially, the patient's current baseline AED should be held at its current dose to limit breakthrough seizures, while the new adjunctive AED is titrated to a presumably effective protective target dose, followed by taper and withdrawal of the baseline AED. The rationale for this measured approach is that abruptly stopping an existing baseline AED increases the risk of breakthrough seizures, while introducing a new adjunctive AED too rapidly can cause an excess of adverse effects.

\section{Pharmacotherapy in PWE}

Among the six families of antidepressant drugs, most of the published data in PWE include open trials with SSRIs, SNRIs, and TCAs and the norepinephrine and dopamine reuptake blocker (e.g., bupropion), which is one of the antidepressant drugs that is not recommended in these patients because of a proconvulsant effect in nonepileptic patients. SSRI are the most widely used classes of antidepressant medications to treat depression in PWE. Other options include SNRI, and, in selected patients, TCAs. In general, the delay of initiating treatment in PWE stems from the belief that antidepressants worsen or trigger seizures.

\section{Do antidepressant drugs cause or worsen seizures?}

Notably, and in contrary to that idea, the actual risk of antidepressant drugs causing or worsening of seizures in PWE is small and should not deter the start of therapy. In a study at the Rush Epilepsy Center, sertraline was found to definitely worsen seizures in only 1 out of 100 patients [73]. In another five patients, a transient increase in seizure frequency was attributed to this antidepressant drug with a probable but not definite causality. On that note, surveyed primary care physicians, neurologists, and psychiatrists chosen from the Ohio State Medical Board registry on the topics of depression in epilepsy, seizures with antidepressant use, have identified fear of increased seizure frequency with antidepressant use as a significant barrier to treatment. There was a clear inverse relationship between the estimated risk of antidepressant-induced seizures and comfort treating depression in epilepsy $(\mathrm{P}=0.02)$, with $52 \%$ of primary care physicians identifying this as a reason for not treating depression in this population. The author called for further education of community physicians and neurologists regarding the importance of treating depression in PWE [74]. On the other hand, bupropion, maprotiline, and amoxapine are the antidepressant drugs with the strongest proconvulsant properties and should be avoided in patients with epilepsy [75].

Furthermore, a recent and very reassuring paper suggests a possible "protective" effect of SSRIs and SNRI in depressed patients: In that study, Alper et al. [76] compared the incidence of seizures between 
depressed patients randomized to placebo and SSRIs, SNRI s and the a2 antagonist mirtazapine in the course of regulatory studies submitted to the FDA. The seizure frequency among patients randomized to placebo was 1501.5 seizures/100,000 years, while that of patients randomized to the antidepressants was 534.8 seizures/100,000 years. Moreover, and contrary to the widespread misbelieve, some investigators have suggested that SSRIs, possibly through its increase extracellular 5-HT, inhibits both focal and generalized seizures in several animal models of generalized epilepsy and focal epilepsy $[77,78]$.

\section{Choice of antidepressant drug}

Clinicians must factor in several variables in the process of choosing a first antidepressant drug among the most commonly used classes SSRIs, SNRIs, and TCAs. The choice of drugs should be individualized according to many variables. Potential pharmacokinetic interactions, patient's age and sex, adverse effect profile of the medication, other comorbid psychiatric and non-psychiatric conditions are among the most important variables the determine the choice of antidepressants.

Pharmacokinetic interactions with concurrent AEDs or any other concurrent medication in patients with other co morbid chronic disease states are among the first important variables that should be considered when choosing a drug or a class of drugs. For example, several drugs of the SSRI family are inhibitors of one or more cytochrome P450 (CYP) isoenzymes; [79] while, citalopram and escitalopram, from that family have a very mild inhibitory effects. This effect becomes extremely important if a patient is using a drug that is metabolized by these enzymes. In addition, it is important to realize that in settings where higher SSRI doses are used, or perhaps in elderly patients who may have reduced clearance of both phenytoin and the SSRI, the potential for a clinically meaningful interaction may be increased. Likewise, several case reports have suggested that SSRIs such as fluoxetine and sertraline have caused increased phenytoin and carbamazepine serum concentrations [80] On the other hand, and unlike the SSRI family, venlafaxine and duloxetine from the SNRI family are unlikely to cause significant interactions with currently available AEDs.

Furthermore, it is worth reporting that most SSRIs, SNRIs, and TCAs are substrates for one or more or the CYP isoenzymes [81], comedication with an enzyme inducing AED is expected to increase their systemic clearance resulting in lower serum concentrations. This effect has been demonstrated in antidepressants, such as, sertraline, paroxetine, citalopram, as well as in most TCAs, requiring an adjustment of their dose (by 25-30\%) to avert recurrence of psychiatric symptoms. In contrast to the enzyme-inducing drugs, the AED sodium valproate can inhibit certain CYP (2C9) and UDPglucuronyltransferase enzymes, and may cause significant increases (50-60\%) in serum concentrations of antidepressants such as amitriptyline or nortriptyline [80].

Finally, the potential for cardiac arrhythmias mediated by a sodium channel blocking effect of high serum concentrations of TCAs needs to be factored in the choice of antidepressant drugs in PWE, given the increased risk of sudden death in PWE. Similarly, the anticholinergic effects of these medications that cause dry mouth, constipation, urinary hesitation/retention, blurred vision, tachycardia, exacerbation of narrow angle glaucoma, problems with memory, and confusion; while, blockade of the alphal adrenoreceptor has been associated with postural hypotension, dizziness, and reflex tachycardia, and can potentiate the effects of antihypertensive drugs. All these negative adverse effects can be quite problematic in patients with diabetes, for example, with its associated widespread neuropathy.

On a separate note, adverse effects of SSRIs and SNRIs have to be considered prior to initiation of treatment. They have been reported in about $20-30 \%$ of patients with primary mood disorders and include anxiety and agitation during the acute phase of treatment, gastrointestinal symptoms (i.e., nausea, abdominal cramping, and diarrhea), and changes in appetite and weight, sexual disturbances, and rarely involuntary movements. Furthermore, SSRIs can compound on a weight gain caused by other AEDs such as valproic acid, gabapentin, pregabalin, and carbamazepine. Among the SNRIs, hypertension is a potential adverse event identified in patients taking venlafaxine, but not duloxetine [82]. Therefore, blood pressures need to be monitored closely and venlafaxine and duloxetine should be used with great caution among patients with a risk or with existing hypertension. This should be an important point to consider, especially in certain part of the world, such as the Gulf region, for example, where chronic conditions such as hypertension, diabetes, and dyslipedemia are quite prevalent [83]. Duloxetine should be used with great care in patients with a history of liver disease and should be avoided in those with glaucoma.

All SSRIs and SNRIs can cause sexual disturbances, although the effect is less frequent with the latter. Accordingly, it is essential for clinicians to investigate the existence of sexual disturbances before starting an SSRI, as these are relatively common among PWE, either, as a direct consequence of the seizure disorder, as an adverse event in response to an AED, or a combination of the two factors, or can be related to other chronic disease states such as diabetes. On that note, citalopram and escitalopram and the SNRIs have been reported to have a lower incidence of sexual adverse events.

Several population-based studies have suggested that SSRIs can cause osteopenia and/or osteoporosis. It is well established that bone disease is quite prevalent in woman with epilepsy either as, a direct effect of the AED, mainly with an enzyme-inducing AEDs that affects the metabolism of vitamin D [84]. This issue becomes of paramount importance in a setting of low vitamin $\mathrm{D}$, especially in certain part of the word, where low vitamin $\mathrm{D}$ is quite prevalent among their women population, which is the case in our Gulf region [85].

\section{Nonpharmacological therapy}

Other options for treatment include cognitive, interpersonal and behavioral therapy, and, in severe cases, electroconvulsive therapy (ECT) can be considered safely in PWE who do not respond to appropriate antidepressant therapy [86]. A randomized trial of an SSRI and cognitive behavior therapy (CBT) in depressed patients without other neurological disorders demonstrated greater efficacy with combined therapy compared to either one alone [87]. A similar trial design for PWE randomizing 140 PWE to either sertaline or CBT for 16 weeks has been recently completed. At the end of the trial, no significant difference in outcome for depressive symptoms remission was observed in the two arms. Moreover, patients whom their depressive symptoms remit had significant improvement in QOL independent of other epilepsy related factors. Similar to the study of Alper et al, no worsening of seizures were observed in patients randomized to sertaline [88].

Finally, in selected cases, one may consider an AED that has an antidepressant and or a mood stabilizing properties to treat both conditions with a single agent. Alternatively, vagal nerve stimulator 
(VNS), which has a unique mechanism of action that would explain its antiepileptic and antidepressants' properties, can also be considered in selected cases [89].

\section{Recommendations}

Worldwide epidemiological studies conquer the high occurrence of depression in people with epilepsy, and the great negative impact this comorbidity has, on these patients at multiple domains. These alerting figures should call on all physicians caring for these patients to screen early for this condition, because early and effective treatment is expected to decrease the duration of depression, increase the patients' compliance with AEDs and, consequently, improve their overall QOL.

\section{Final thoughts}

Future epidemiological studies should aim to address the unanswered question of the risk factors to develop depression in epilepsy patients and the impact of such association on selecting the most optimal treatment strategies. To date, there have been no clinical studies that have investigated whether antidepressant treatment has any disease-modifying effects on the epilepsy itself (as opposed to antiseizure or pro-seizure effects). Undertaking such a study would be logistically difficult, requiring long-term treatment and follow-up, and appropriately matched placebo-treated controls. This planned study needs to address the various manifestation of epilepsy progression, such as seizure frequency, AED resistance, neuropsychiatric and neurocognitive deficits and structural brain changes.

Additionally, future studies need to investigate the effects of chronic SSRI exposure, studying the effects on seizures at clinically relevant time points including prior to seizure onset, at onset and, at the chronic stages. That trial should investigate the effects of antidepressant use on mediators of epileptogenesis and how this phenomenon can be altered with antidepressant treatment.

\section{References}

1. Téllez-Zenteno JF, Matijevic S, Wiebe S (2005) Somatic comorbidity of epilepsy in the general population in Canada. Epilepsia 46: 1955-1962.

2. Ettinger A, Reed M, Cramer J (2004) Depression and comorbidity in community based patients with epilepsy or asthma. Neurology 63:10081014

3. Gaitatzis A, Carroll K, Majeed A, Sander JW (2004) The epidemiology of the comorbidity of epilepsy in the general population. Epilepsia 45:1613-1622.

4. Ettinger AB, Reed ML, Goldberg JF, Hirschfeld RM (2005) Prevalence of bipolar symptoms in epilepsy vs other chronic health disorders. Neurology 65: 535-540.

5. Tellez-Zenteno JF, Patten SB, Jetté N, Williams J, Wiebe S (2007) Psychiatric comorbidity in epilepsy: a population-based analysis. Epilepsia 48: 2336-2344.

6. Jones JE, Hermann BP, Barry JJ, Gilliam FG, Kanner AM, et al. (2003) Rates and risk factors for suicide, suicidal ideation, and suicide attempts in chronic epilepsy. Epilepsy Behav 4 Suppl 3: S31-38.

7. Pompili M, Girardi P, Tatarelli R (2006) Death from suicide versus mortality from epilepsy in the epilepsies: a meta-analysis. Epilepsy Behav 9: 641-648.

8. Christensen J, Vestergaard M, Mortensen PB, Sidenius P, Agerbo E (2007) Epilepsy and risk of suicide: a population-based case-control study. Lancet Neurol 6: 693-698.

9. Mazza M, Bria P, Mazza S (2007) Depression and suicide in epilepsy: fact or artefact? J Neurol Sci 260: 300-301.
10. Robertson MM, Trimble MR, Townsend HR (1987) Phenomenology of depression in epilepsy. Epilepsia 28: 364-372.

11. Jacoby A, Baker GA, Steen N, Potts P, Chadwick DW (1996) The clinical course of epilepsy and its psychosocial correlates: findings from a U.K. Community study. Epilepsia 37: 148-161.

12. Mendez MF, Cummings JL, Benson DF (1986) Depression in epilepsy. Significance and phenomenology. Arch Neurol 43: 766-770.

13. O'Donoghue MF, Goodridge DM, Redhead K, Sander JW, Duncan JS (1999) Assessing the psychosocial consequences of epilepsy: a community-based study. Br J Gen Pract 49: 211-214.

14. Seminario NA, Farias ST, Jorgensen J, Bourgeois JA, Seyal M (2009) Determination of prevalence of depression in an epilepsy clinic using a brief DSM-IV-based self-report questionnaire. Epilepsy Behav 15: 362-366.

15. El Hammasi, Raouf M, Shahrour T, et al. (2015) Prevalence of Depression and Anxiety among patients with epilepsy attending the epilepsy clinic at Sheikh Khalifa Medical City. 168th annual meeting and at the 68th American Academy of Neurology Meeting.

16. Fiest KM, Dykeman J, Patten SB, Wiebe S, Kaplan GG, et al. (2013) Depression in epilepsy: a systematic review and meta-analysis. Neurology 80: 590-599.

17. Beghi E, Spagnoli P, Airoldi L, Fiordelli E, Appollonio I, et al. (2002) Emotional and affective disturbances in patients with epilepsy. Epilepsy Behav 3: 255-261.

18. Lehrner J, Kalchmayr R, Serles W, Olbrich A, Pataraia E, et al. (1999) Health-related quality of life (HRQOL), activity of daily living (ADL) and depressive mood disorder in temporal lobe epilepsy patients. Seizure 8: 88-92.

19. Perrine K, Hermann BP, Meador KJ, Vickrey BG, Cramer JA, et al. (1995) The relationship of neuropsychological functioning to quality of life in epilepsy. Arch Neurol 52: 997-1003.

20. Gilliam F, Kuzniecky R, Faught E, Black L, Carpenter G, et al. (1997) Patient-validated content of epilepsy-specific quality-of-life measurement. Epilepsia 38: 233-236.

21. Boylan LS, Flint LA, Labovitz DL, Jackson SC, Starner K, et al. (2004) Depression but not seizure frequency predicts quality of life in treatmentresistant epilepsy. Neurology 62: 258-261.

22. Cramer JA, Blum D, Reed M, Fanning K; Epilepsy Impact Project Group (2003) The influence of comorbid depression on quality of life for people with epilepsy. Epilepsy Behav 4: 515-521.

23. Hamid H, Blackmon K, Cong X, Dziura J, Atlas LY, et al. (2014) Mood, anxiety, and incomplete seizure control affect quality of life after epilepsy surgery. Neurology 82: 887-894.

24. Harris EC, Barraclough B (1997) Suicide as an outcome for mental disorders: a meta-analysis. Br J Psychiatry. 170:205-228.

25. Cramer JA, Blum D, Fanning K, Reed M; Epilepsy Impact Project Group (2004) The impact of comorbid depression on health resource utilization in a community sample of people with epilepsy. Epilepsy Behav 5: 337-342.

26. Hitiris N, Mohanraj R, Norrie J, Sills GJ, Brodie MJ (2007) Predictors of pharmacoresistant epilepsy. Epilepsy Res 75: 192-196.

27. Kanner AM, Byrne R, Chicharro A, Wuu J, Frey M (2009) A lifetime psychiatric history predicts a worse seizure outcome following temporal lobectomy. Neurology 72: 793-799.

28. Kanner AM (2005) Depression in epilepsy: a neurobiologic perspective. Epilepsy Curr 5: 21-27.

29. Kanner AM, Palac S (2000) Depression in epilepsy: a common but often unrecognized comorbid malady. Epilepsy Behav 1: 37-51.

30. Mazarati A, Shin D, Auvin S, Caplan R, Sankar R (2007) Kindling epileptogenesis in immature rats leads to persistent depressive behavior. Epilepsy Behav 10: 377-383.

31. Drevets WC, Frank E, Price JC, et al. (1999) PET imaging of serotonin 1A receptor binding in depression. Biol Psychiatry 46:1375-1387. 
32. Jobe PC, Dailey JW, Wernicke JF (1999) A noradrenergic and serotonergic hypothesis of the linkage between epilepsy and affective disorders. Crit Rev Neurobiol 13: 317-356.

33. Lothe A, Didelot A, Hammers A, Costes N, Saoud M, et al. (2008) Comorbidity between temporal lobe epilepsy and depression: a [18F]MPPF PET study. Brain 131: 2765-2782.

34. Theodore WH, Giovacchini G, Bonwetsch R, Bagic A, Reeves-Tyer P, et al. (2006) The effect of antiepileptic drugs on 5-HT-receptor binding measured by positron emission tomography. Epilepsia 47: 499-503.

35. Forsgren L, Nyström L (1990) An incident case-referent study of epileptic seizures in adults. Epilepsy Res 6: 66-81.

36. Hesdorffer DC, Hauser WA, Annegers JF, Cascino G (2000) Major depression is a risk factor for seizures in older adults. Ann Neurol 47: 246-249.

37. Hesdorffer DC, Hauser WA, Olafsson E, Ludvigsson P, Kjartansson O (2006) Depression and suicide attempt as risk factors for incident unprovoked seizures. Ann Neurol 59: 35-41.

38. Adelöw C, Andersson T, Ahlbom A, Tomson T (2012) Hospitalization for psychiatric disorders before and after onset of unprovoked seizures/ epilepsy. Neurology 78: 396-401

39. Lewis A (1934) Melancholia: a historical review. J Mental Sci. 80:1-42.

40. Sheline YI (2003) Neuroimaging studies of mood disorder effects on the brain. Biol Psychiatry 54: 338-352.

41. Sheline YI, Gado MH, Kraemer HC (2003) Untreated depression and hippocampal volume loss. Am J Psychiatry 160: 1516-1518.

42. Quiske A, Helmstaedter C, Lux S, Elger CE (2000) Depression in patients with temporal lobe epilepsy is related to mesial temporal sclerosis. Epilepsy Res 39: 121-125.

43. Gilliam FG, Maton BM, Martin RC, Sawrie SM, Faught RE, et al. (2007) Hippocampal $1 \mathrm{H}$-MRSI correlates with severity of depression symptoms in temporal lobe epilepsy. Neurology 68: 364-368.

44. Mula M, Trimble MR, Sander JW (2003) The role of hippocampal sclerosis in topiramate-related depression and cognitive deficits in people with epilepsy. Epilepsia 44: 1573-1577

45. Mula M, Trimble MR, Sander JW (2007) Are psychiatric adverse events of antiepileptic drugs a unique entity? A study on topiramate and levetiracetam. Epilepsia 48: 2322-2326.

46. Lambert MV, Robertson MM (1999) Depression in epilepsy: etiology, phenomenology, and treatment. Epilepsia 40 Suppl 10: S21-47.

47. Shamim S, Hasler G, Liew C, Sato S, Theodore WH (2009) Temporal lobe epilepsy, depression, and hippocampal volume. Epilepsia 50: 1067-1071.

48. Ketter TA, Malow BA, Flamini R, White SR, Post RM, et al. (1994) Anticonvulsant withdrawal-emergent psychopathology. Neurology 44: 55-61.

49. Devinsky O, Barr WB, Vickrey BG, Berg AT, Bazil CW, et al. (2005) Changes in depression and anxiety after resective surgery for epilepsy. Neurology 65: 1744-1749.

50. Waxman SG, Geschwind N (1974) Hypergraphia in temporal lobe epilepsy. Neurology 24: 629-636.

51. Blumer D (1999) Evidence supporting the temporal lobe epilepsy personality syndrome. Neurology 53: S9-12.

52. Devinsky O, Najjar S (1999) Evidence against the existence of a temporal lobe epilepsy personality syndrome. Neurology 53: S13-25.

53. Benson DF (1991) The Geschwind syndrome. Adv Neurol 55: 411-421.

54. Van Elst LT, Krishnamoorthy ES, Bäumer D, Selai C, von Gunten A, et al. (2003) Psychopathological profile in patients with severe bilateral hippocampal atrophy and temporal lobe epilepsy: evidence in support of the Geschwind syndrome? Epilepsy and Behavior 4: 291-297.

55. Blumer D, Altshuler LL (1998) Affective disorders, Epilepsy: A Comprehensive Textbook. (2ndedn), Lippincott-Raven, Philadelphia, USA.
56. Mula M, Jauch R, Cavanna A, Gaus V, Kretz R, et al. (2010) Interictal dysphoric disorder and periictal dysphoric symptoms in patients with epilepsy. Epilepsia 51: 1139-1145.

57. Savard G, Andermann LF, Reutens D, Andermann F (1998) Epilepsy, surgical treatment and postoperative psychiatric Complications: A reevaluation of the evidence, Forced Normalization and Alternative Psychosis of Epilepsy Petersfield. Wrightson Biomedical, UK.

58. Gilliam F (2002) Optimizing health outcomes in active epilepsy. Neurology 58: S9-20.

59. Gilliam FG, Barry JJ, Hermann BP, Meador KJ, Vahle V, et al. (2006) Rapid detection of major depression in epilepsy: a multicentre study. Lancet Neurol 5: 399-405.

60. Rampling J, Mitchell AJ, Von Oertzen T, Docker J, Jackson J, et al. (2012) Screening for depression in epilepsy clinics. A comparison of conventional and visual-analog methods. Epilepsia 53: 1713-1721.

61. Rathore JS, Jehi LE, Fan Y, Patel SI, Foldvary-Schaefer N, et al. (2014) Validation of the Patient Health Questionnaire-9 (PHQ-9) for depression screening in adults with epilepsy. Epilepsy Behav 37: 215-220.

62. http://www.fda.gov/ohrms/dockets/ac/08/briefing/2008-4372b1-01FDA.pdf

63. Alsaadi T (2010) Neuropsychiatric issues in patients with epilepsy: focus on depression. Therapy 7: 507-515.

64. Hesdorffer DC, Kanner AM (2009) The FDA alert on suicidality and antiepileptic drugs: Fire or false alarm? Epilepsia 50: 978-986.

65. Gibbons RD, Hur K, Brown CH, Mann JJ (2009) Relationship between antiepileptic drugs and suicide attempts in patients with bipolar disorder. Arch Gen Psychiatry 66: 1354-1360.

66. Olesen JB, Hansen PR, Erdal J, Abildstrøm SZ, Weeke P, et al. (2010) Antiepileptic drugs and risk of suicide: a nationwide study. Pharmacoepidemiol Drug Saf 19: 518-524.

67. Patorno E, Bohn RL, Wahl PM, Avorn J, Patrick AR, et al. (2010) Anticonvulsant medications and the risk of suicide, attempted suicide, or violent death. JAMA 303: 1401-1409.

68. Robertson MM, Trimble MR (1985) The treatment of depression in patients with epilepsy. A double-blind trial. J Affect Disord 9: 127-136.

69. Maguire MJ, Weston J, Singh J, Marson AG (2014) Antidepressants for people with epilepsy and depression. Cochrane Database Syst Rev 12: CD010682.

70. http://www.nice.org.uk/page.aspx?o=235213

71. Piedad J, Rickards H, Besag FM, Cavanna AE (2012) Beneficial and adverse psychotropic effects of antiepileptic drugs in patients with epilepsy: a summary of prevalence, underlying mechanisms and data limitations. CNS Drugs 26: 319-335.

72. Mula M, Hesdorffer DC, Trimble M, Sander JW (2009) The role of titration schedule of topiramate for the development of depression in patients with epilepsy. Epilepsia 50: 1072-1076.

73. Kanner AM, Kozak AM, Frey M (2000) The Use of Sertraline in Patients with Epilepsy: Is It Safe? Epilepsy Behav 1: 100-105.

74. Cotterman-Hart S (2010) Depression in epilepsy: why aren't we treating? Epilepsy Behav 19: 419-421.

75. Pisani F, Spina E, Oteri G (1999) Antidepressant drugs and seizure susceptibility: from in vitro data to clinical practice. Epilepsia $40 \mathrm{Suppl}$ 10: S48-56.

76. Alper K, Schwartz KA, Kolts RL, Khan A (2007) Seizure incidence in psychopharmacological clinical trials: an analysis of Food and Drug Administration (FDA) summary basis of approval reports. Biol Psychiatry 62: 345-354.

77. Hamid H, Kanner AM (2013) Should antidepressant drugs of the selective serotonin reuptake inhibitor family be tested as antiepileptic drugs? Epilepsy Behav 26: 261-265.

78. Vermoesen K, Massie A, Smolders I, Clinckers R (2012) The antidepressants citalopram and reboxetine reduce seizure frequency in rats with chronic epilepsy. Epilepsia 53: 870-878. 
Citation: Alsaadi T, Shahrour TM (2015) Depressive Disorders in Patients with Epilepsy: Underdiagnosed and Appropriately Managed?. Brain Disord Ther 4: 163. doi:10.4172/2168-975X.1000163

Page 9 of 9

79. Trimble MR, Mula M (2005) Antiepileptic drug interactions in patients requiring psychiatric drug treatment, Antiepileptic drugs. Combination therapy and interactions. Cambridge University Press, Cambridge, U.K.

80. Nelson MH, Birnbaum AK, Remmel RP (2001) Inhibition of phenytoin hydroxylation in human liver microsomes by several selective serotonin re-uptake inhibitors. Epilepsy Res 44: 71-82.

81. Patsalos PN, Perucca E (2003) Clinically important drug interactions in epilepsy: interactions between antiepileptic drugs and other drugs. Lancet Neurol 2: 473-481.

82. Kent JM, Gorman JM (1998) Venlafaxine, Textbook of psychopharmacology. (2ndedn), American Psychiatric Association Press, Washington, USA.

83. Alhyas L, McKay A, Balasanthiran A, Majeed A (2011) Prevalences of overweight, obesity, hyperglycaemia, hypertension and dyslipidaemia in the Gulf: systematic review. JRSM Short Rep 2: 55.

84. Diem SJ, Blackwell TL, Stone KL, Yaffe K, Haney EM, et al. (2007) Use of antidepressants and rates of hip bone loss in older women: the study of osteoporotic fractures. Arch Intern Med 167: 1240-1245.
85. Al-Daghri NM, Al-Attas OS, Alokail MS, Alkharfy KM, El-Kholie E, et al. (2012) Increased vitamin D supplementation recommended during summer season in the gulf region: a counterintuitive seasonal effect in vitamin D levels in adult, overweight and obese Middle Eastern residents. Clin Endocrinol (Oxf). Mar; 76:346-50.

86. Krahn LE, Rummans TA, Peterson GC, Cascino GD, Sharbrough FW (1993) Electroconvulsive Therapy for Depression After Temporal Lobectomy for Epilepsy. Convuls Ther 9: 217-219.

87. Keller MB, McCullough JP, Klein DN, Arnow B, Dunner DL, et al. (2000) A comparison of nefazodone, the cognitive behavioral-analysis system of psychotherapy, and their combination for the treatment of chronic depression. N Engl J Med 342: 1462-1470.

88. Gilliam F, Black kj, Carter J (2009) Depression and health outcomes in epilepsy: a randomized trial. Neurology 72: A261-262.

89. Rush AJ, Marangell LB, Sackeim HA, George MS, Brannan SK, et al. (2005) Vagus nerve stimulation for treatment-resistant depression: a randomized, controlled acute phase trial. Biol Psychiatry 58: 347-354. 